DEVELOPMENT

\section{Go with the flow}

The intimate relationship between the nervous and circulatory systems is highlighted by two recent studies.

In peripheral tissues, nerves and blood vessels enjoy a close anatomical relationship. Nerves depend on this association to satisfy their need for oxygen and nutrients, while regulating the functional properties of blood vessels by vasoconstriction and vasodilation. This neurovascular relationship is dependent on the common branching patterns of nerves and blood vessels. But how is this patterning achieved? Mukouyama et al. examined the expression of arteryspecific markers in embryonic mouse skin, and found that arteries, but not veins, were aligned with peripheral nerves. Mutations that eliminated sensory nerves and/or Schwann cells prevented normal arteriogenesis, whereas those that disorganized nerves altered the trajectories of arteries, which followed the misrouted axons. Moreover, the authors showed that arteriogenesis was initiated only after the association of smaller blood vessels with nerves. In vitro, sensory neurons or Schwann cells could induce the expression of arterial markers in embryonic endothelial cells, and vascular endothelial growth factor (VEGF) was necessary and sufficient to cause this induction. In vivo, VEGF expression was shown to be markedly reduced in mutants that lacked Schwann cells. So, peripheral nerves seem to govern the pattern of blood vessel branching and arterial differentiation in the skin by the secretion of VEGF from sensory fibres and/or Schwann cells. By directing the differentiation and patterning of arteries, nerves might secure a local source of neurotrophic factors for subsequent growth.

The second study provides an example of coordinated angiogenesis and neurogenesis in the adult forebrain. In the higher vocal centre (HVC) of the adult songbird neostriatum, neurogenesis continues throughout life, and testosterone can induce neuronal recruitment into this

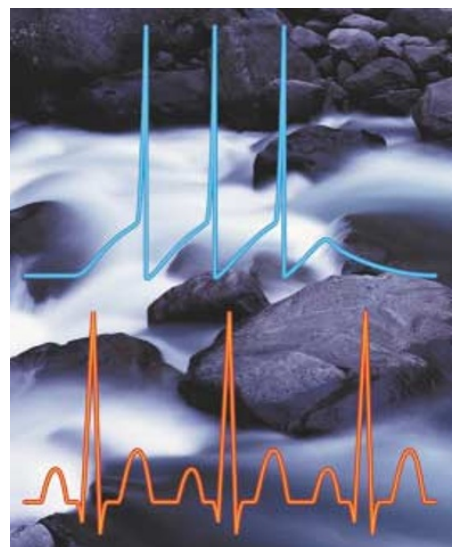

structure. Louissaint et al. explored the possibility that testosteroneinduced angiogenesis contributes to HVC neuronal addition. They found that testosterone upregulated VEGF and its endothelial receptor, VEGFR2, in the HVC, resulting in a surge of local angiogenesis. In vitro, isolated HVC endothelial cells produced brain-derived neurotrophic factor (BDNF) in a testosterone-dependent fashion; furthermore, BDNF could promote the outgrowth of neurons from the cultured HVC ventricular zone. In vivo, the levels of HVC BDNF rose after testosterone treatment more than a week after the induction of VEGF and its receptor - and this increase was associated with the recruitment of neurons into the HVC. Finally, the authors showed that both angiogenesis and neuronal addition were greatly attenuated by the inhibition of VEGFR2. So, gonadal steroids seem to act on the microvascular bed to promote neuronal recruitment in the adult brain.

These studies converge on the importance of the local vasculature for nervous system development and function, and point to the reciprocal nature of the relationship between nerves and blood vessels.

Rebecca Craven

\section{(1) References and links} ORIGINAL RESEARCH PAPER Mukouyama, Y.-S. et al. Sensory nerves determine the pattern of arterial differentiation and blood vessel branching in the skin. Cell 109, 693-705 (2002) | Louissaint, A. Jr et al. Coordinated interaction of neurogenesis and angiogenesis in the adult songbird brain. Neuron 34, 945-960 (2002)

\section{IN BRIEF}

\author{
DEVELOPMENT
}

Cyclin-dependent kinase 5 phosphorylates disabled 1 independently of reelin signaling.

Keshvara, L. et al. J. Neurosci. 22, 4869-4877 (2002)

Reelin and cyclin-dependent kinase 5 (Cdk5) have a crucial role in the control of neuronal migration during development; the independent disruption of these signalling pathways leads to comparable neuroanatomical defects. Keshvara et al. report that Cdk5 phosphorylates disabled 1 , a molecule associated with reelin signalling. This observation is the first direct evidence of biochemical interactions between the two pathways.

\section{AGEING}

Degenerative age changes in white matter connectivity visualized in vivo using magnetic resonance imaging.

Davatzikos, C. \& Resnick, S. M. Cereb. Cortex 12, 761-771 (2002)

A cross-sectional and longitudinal imaging analysis of aged brains showed white matter abnormalities, particularly in the left hemisphere. More importantly, a comparison of these abnormalities with the volumetric changes that are commonly observed in ageing showed that the two measures were unrelated, and that the alterations in the white matter signal correlated more strongly with age. So, this kind of change in the white matter might be a more sensitive marker of preclinical degeneration states.

\section{AXON GUIDANCE}

Axonal protein synthesis provides a mechanism for localized regulation at an intermediate target.

Brittis, P. A. et al. Cell 1 July 2002 (doi:10.1016/\$0092867402008139)

As local receptor upregulation might affect growth cone responsiveness to guidance cues, the authors explored whether axonal protein synthesis participates in this upregulation. They found that severed axons synthesize proteins and export them to the membrane. Moreover, a sequence in the $3^{\prime}$ untranslated region of the ephrin EphA2 mRNA targets reporter genes to distal axon segments.

\section{SYNAPTIC TRANSMISSION}

Synaptotagmins I and IV promote transmitter release independently of $\mathrm{Ca}^{2+}$ binding in the $\mathrm{C}_{2} \mathrm{~A}$ domain.

Robinson, I. M. et al. Nature 7 July 2002 (doi:10.1038/nature00915)

The $\mathrm{C}_{2} \mathrm{~B} \mathrm{Ca}^{2+}$-binding motif of synaptotagmin is required for synaptic transmission in vivo.

Mackler, J. M. et al. Nature 7 July 2002 (doi:10.1038/nature00846)

Synaptotagmin I seems to serve as the $\mathrm{Ca}^{2+}$ sensor during synaptic vesicle exocytosis. This protein has two $\mathrm{C}_{2}$ domains that bind $\mathrm{Ca}^{2+}$, and these two genetic studies show that the $\mathrm{C}_{2} \mathrm{~B}$, but not the $\mathrm{C}_{2} \mathrm{~A}$, domain is important for vesicle fusion in Drosophila. In addition, Robinson et al. report that synaptotagmin IV can substitute for synaptotagmin I, indicating that it promotes release, rather than inhibiting it (as was previously thought). 